\title{
Recent status and results of the Dark Matter Particle Explorer
}

\section{Xiang $\mathbf{L i}^{a, b, *}$ on behalf of the DAMPE Collaboration}

(a complete list of authors can be found at the end of the proceedings)

${ }^{a}$ Key Laboratory of Dark Matter and Space Astronomy, Purple Mountain Observatory, Chinese Academy of Sciences, Nanjing 210023, China

${ }^{b}$ School of Astronomy and Space Science, University of Science and Technology of China, Hefei 230026, China

E-mail: xiangli@pmo.ac.cn

The DArk Matter Particle Explorer (DAMPE) is a satellite-borne, calorimetric type, high-energyresolution space cosmic ray and gamma-ray detector. It was launched in December 2015 and has been stably operating for more than five years. Its three major scientific objectives are dark matter indirect detection, cosmic ray physics and gamma-ray astronomy. Precise measurements of the all-electron, proton and Helium spectra in wide energy ranges have been obtained, shedding new light on the research of cosmic ray physics and dark matter properties. We will also present the current status of the mission and its recent physical results.

$37^{\text {th }}$ International Cosmic Ray Conference (ICRC 2021)

July 12 th - 23rd, 2021

Online - Berlin, Germany

\footnotetext{
*Presenter
} 


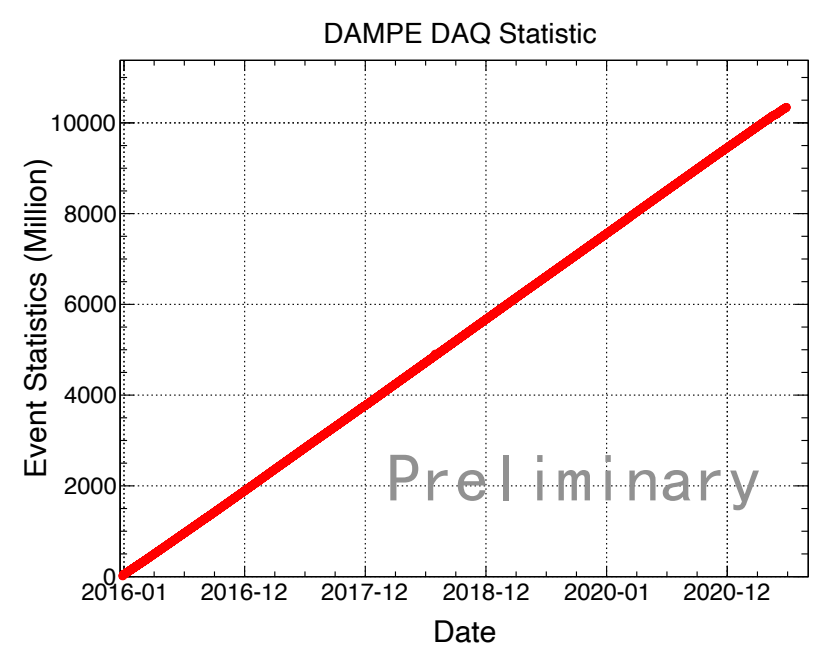

Figure 1: The number of events accumulated by DAMPE.

\section{Introduction}

The Dark Matter Particle Explorer (DAMPE, also known as Wukong in China) is a satelliteborne, calorimetric type, high-energy particle detector dedicated to the indirect detection of dark matter (DM) in space and astrophysical studies [1,2]. As one of the four scientific space science missions within the framework of the strategic priority science and technology projects in space science of the Chinese Academy of Sciences, it was launched to a $500 \mathrm{~km}$ Sun-synchronous orbit on Dec. 17th, 2015 from the Jiuquan Satellite Launch Center. The major scientific objectives addressed by DAMPE include probing the dark matter via the detection of high-energy electrons/positrons and gamma rays, understanding the origin, acceleration and propagation of cosmic rays in the Milky Way, and studying the gamma-ray astronomy. During the 5.5 years of on-orbit operation, both the satellite and the payload work perfectly. So far DAMPE has surveyed the whole sky for over 11 times and obtained more than 10 billion high-energy events as of June 30th, 2021 (Fig. 1).

The DAMPE detector consists of four sub-detectors (Fig. 2). They are the Plastic Scintillator strip Detector (PSD; [3]), the Silicon-Tungsten tracKer-converter (STK; [4]), the BGO imaging calorimeter [5], and the NeUtron Detector (NUD; [6]). The PSD measures the charge of incident particles via the ionization effect, and is used as veto of charged CRs for $\gamma$-ray detection. The STK measures the trajectory and also charge of particles. The BGO is to measure the energy of incident particles and provide electron-proton discrimination based on shower morphologies. The NUD provides additional electron-proton discrimination through the neutron content difference between hadronic and electromagnetic showers. The DAMPE detector is optimized for observations of $\mathrm{CR}$ electrons/positrons (hereinafter CREs) and $\gamma$-rays up to $10 \mathrm{TeV}$ energies with unprecedentedly high energy resolution and electron-proton discrimination capability, thanks to the design of a full-absoption, thick ( 32 radiation lengths) calorimeter. It can thus improve significantly the measurements of the CRE spectrum and the sensitivity of searching for DM particles. The on-orbit calibration shows that each sub-detector works very stably since the lanuch $[7,8]$. 


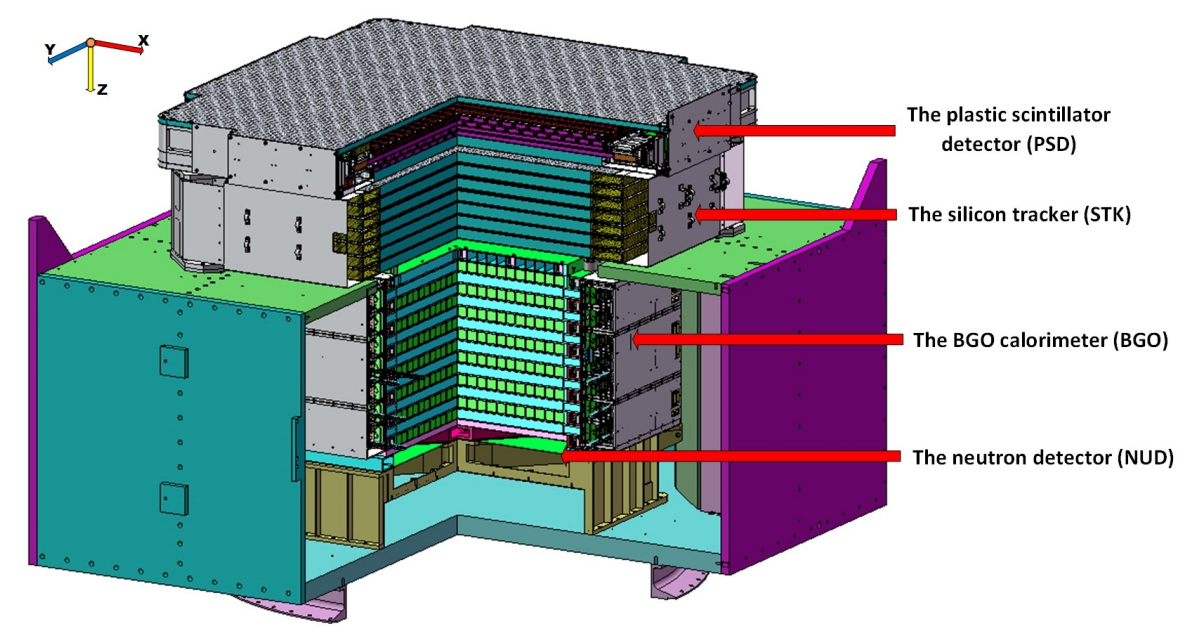

Figure 2: Schematic view of the DAMPE detector [2].

\section{On-orbit calibration and performance}

DAMPE enables several modes for data acquisition (DAQ), including three "calibration modes" for the calibration of the electronics linearity and pedestal, and the "observation mode" for science data taking. Calibrations other than the electronics linearity and pedestal, including the minimum ionization particle (MIP) response, light attenuation, dynode ratios, detector alignment etc. are done with data taken in the "observation mode". In each orbit, the DAQ system switches from the "observation mode" to one "calibration mode" twice — each with about 40 seconds — when the satellite is at a latitude of $20^{\circ} \mathrm{N}$. The details about the calibration of each sub-detector can be found in [7].

The PSD detector is mainly used for charge detection. The particle charge is proportional to the square root of the deposit energy in the PSD bar. For proton MIP events, the energy deposition in the PSD is about $2 \mathrm{MeV} \mathrm{cm}^{-1}$. After a series of corrections such as the attenuation of scintillation light in the PSD strip [9], the alignment of the PSD strips [10], the equalization of different strips, as well as the quenching effect, we finally get high-resolution measurement of particle charges [11]. Fig. 4 in [11] shows the charge spectrum based on two years of flight data of DAMPE. Gaussian fittings show that the charge resolution is about $0.2 e$ for $\mathrm{CNO}$ and $0.3 e$ for $\mathrm{Fe}$. The PSD detector is also used as the anticoincidence detector for $\gamma$-ray detection. The charge-detection power of each PSD strip is always greater than $99.7 \%$ [12], and the overall charge-rejection power of the whole PSD is greater than $10^{5}$.

The STK is primarily used to measure the trajectory as well as the charge (for $Z<8$ ) of particles. $\gamma$-ray photons can convert into a pair of electron and positron in the tungsten plates inserted into the STK, leaving also track imprint in the STK. The direction measurement basically uses the information of hit positions, while the charge measurement uses the signal strength along the trajectory. The calibration of the STK includes the pedestal and noise evaluation, the equalization of the VA140 chips, and the alignment of silicon sensors [13-15]. The spatial resolution of the STK is about $50 \mu \mathrm{m}$ after the alignment procedure [13]. Monte Carlo (MC) simulations show that the angular resolution of normal incident photons is about $1.2^{\circ}\left(0.1^{\circ}\right)$ at $1(100) \mathrm{GeV}$ [2]. 
The BGO calorimeter is to measure the particle energy, to discriminate CREs from protons, and to measure the trajectory of a particle though with limited resolution. The BGO calibration includes the pedestal calibration, the MIP response, the PMT dynode ratio evaluation, the light attenuation length calculation, and the trigger threshold determination [16]. The beam tests verified that the energy resolution of the BGO reaches $\sim 1 \%$ for CREs when $E>100 \mathrm{GeV}$ [17].

The NUD is to measure the secondary neutrons produced by the particle shower in the BGO. The pedestals of NUD are calibrated using periodic trigger events during the dedicated "pedestal calibration mode" every day. The gate open for neutron signal integration is set to be $2.5 \mu \mathrm{s}$ to suppress the charged secondary particles.

The satellite travels through the South Atlantic Anomaly (SAA) region nearly 7 times per day. The very intensive particle hit rate within the SAA region may induce severe pileup effects, which hinders science analyses. The trigger rates stored in the house keeping data are used to determine the boundary of the SAA region $[7,18]$. Photon events from bright $\gamma$-ray sources are used to correct the boresight alignment [19].

Fig. 3 shows the pedestal variation of the four sub-detectors of DAMPE since its launch, which indicates its good stability.
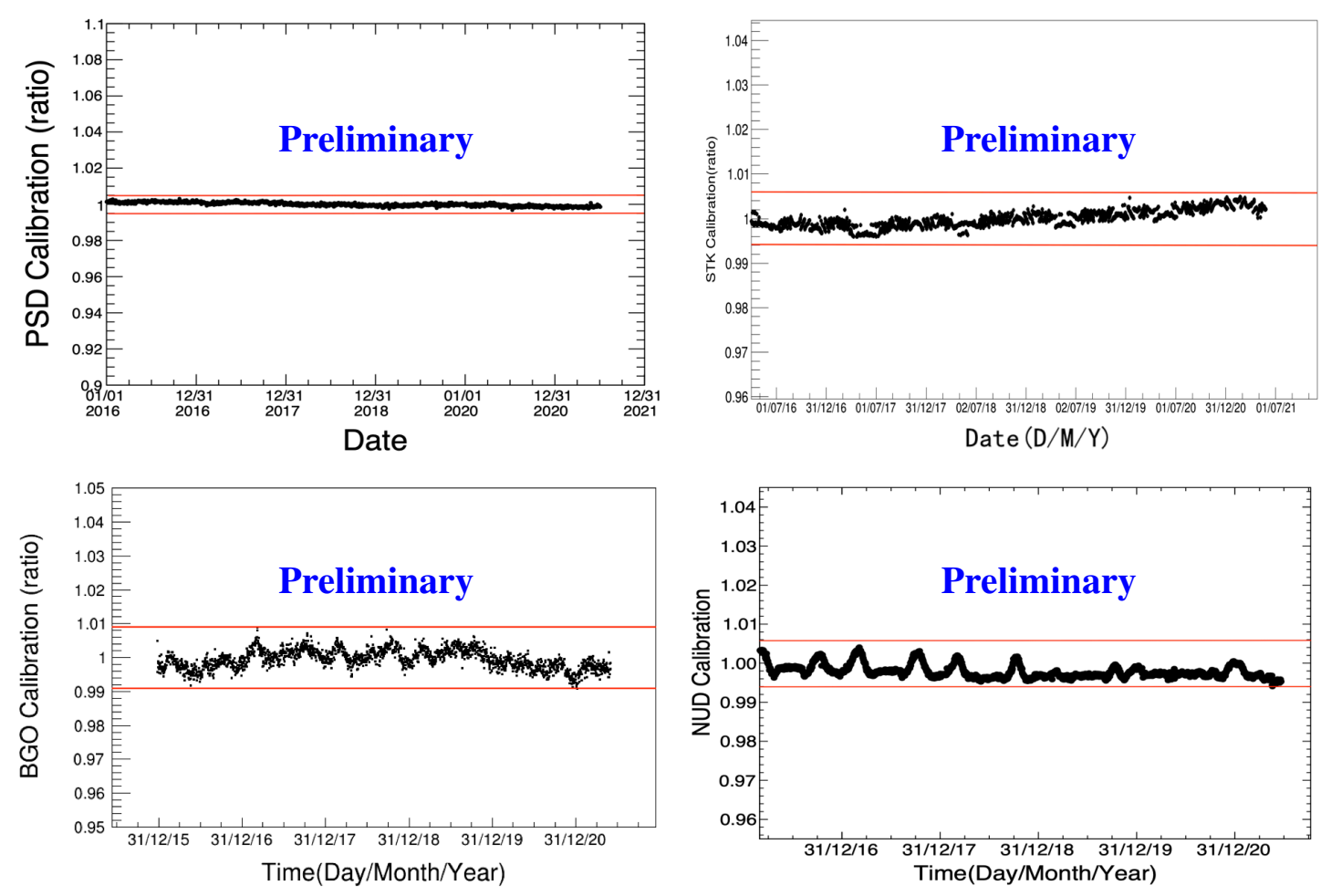

Figure 3: The pedestal variation during the 5.5-year operation of DAMPE.

\section{CRE spectrum}

High energy CREs are ideal probe of nearby CR sources due to their limited propagation distance. They are also sensitive to search for the annihilation or decay of DM particles. The 
observations of CREs are challenging because of the high fluxes from the proton background. Therefore a high rejection power of $\mathrm{CR}$ protons is crucial to reliably identifying CREs. The DAMPE experiment mainly uses shower morphologies in the BGO calorimeter to discriminate CREs from protons (heavier nuclei can be significantly rejected through charge measurement additionally) [20]. A two-parameter method based on the quantification of the transverse and longitudinal distributions of showers is developed. The transverse spread of a shower is described by an energy-dependent root-mean-square (RMS) value of hit positions:

$$
\mathrm{RMS}_{i}=\sqrt{\frac{\sum_{j}\left(x_{j, i}-x_{c, i}\right)^{2} E_{j, i}}{\sum_{j} E_{j, i}}},
$$

where $x_{j, i}$ and $E_{j, i}$ are coordinate and deposit energy of the $j$ th bar in the $i$ th layer, and $x_{c, i}$ is the coordinate of the shower center of the $i$ th layer. The longitudinal distribution parameter uses the energy fraction of the last BGO layer $\left(\mathcal{F}_{\text {last }}\right)$ to describe the tail of a shower. A joint parameter, $\zeta=\mathcal{F}_{\text {last }} \times\left(\sum_{i} \mathrm{RMS}_{i} / \mathrm{mm}\right)^{4} /\left(8 \times 10^{6}\right)$, is employed to select CRE candidates. We find that when setting $\zeta \leq 8.5$, a very clean CRE sample with only $\sim 2 \%$ contamination of the proton background can be obtained for $\mathrm{TeV}$ energies. A few independent methods are also applied to distinguish CRE from CR protons, giving similar results as this two-parameter method. More details can be found in [21].

Using 530 days of flight data, we measure the CRE spectrum from $25 \mathrm{GeV}$ to $4.6 \mathrm{TeV}$ with high precision [21]. Figure 4 shows the CRE spectrum measured by DAMPE, and that by AMS-02 [22], Fermi-LAT [23], CALET [24], and H.E.S.S. [25, 26]. For the first time, the DAMPE result reveals a clear spectral break of the CRE spectrum at $\sim 0.9 \mathrm{TeV}$ at a $\sim 6.6 \sigma$ level, with the spectral index changing from -3.1 to -3.9 . Previously, the H.E.S.S. data together with low energy measurements by other experiments showed only weak evidence for such a break, with relatively large systematic uncertainties (shown by the shaded band in Fig. 4).

The DAMPE result has important implications on the understanding of the modeling of CREs. The wide energy range coverage and high precision of the DAMPE spectrum can significantly improve the constraints on the model parameters to fit the positron excess, either for astrophysical models or the DM annihilation or decay [27]. The spectral break may naturally reflect the discretness of the source distribution due to the fast cooling of TeV CREs [28]. Another interpretation ascribes the softening to the confinement and cooling of CREs during the acceleration stage [29].

The uncertainties of the CRE spectrum by DAMPE include statistical and systematical parts. A good news is that the measurement above $\sim \mathrm{TeV}$ region is still dominated by the statistical uncertainties (See Fig. 5), which indicates the potential ability of DAMPE for CRE measurements with the data accumulating in the future.

\section{CR Nuclei spectra}

The current precise measurements of the spectra of nuclei come from the magnetic spectrometer experiments, such as PAMELA [30] and AMS-02 [22, 31, 32], but due to the limited magnetic field strength, their measurements are limited to below a few $\mathrm{TeVs} / \mathrm{n}$. The balloon-borne experiments such as ATIC [33] and CREAM [34, 35], and satellite experiment NUCLEON [36, 37] extend the measurements to $100 \mathrm{TeV}$ energies, but with large statistical and/or systematic uncertainties. 


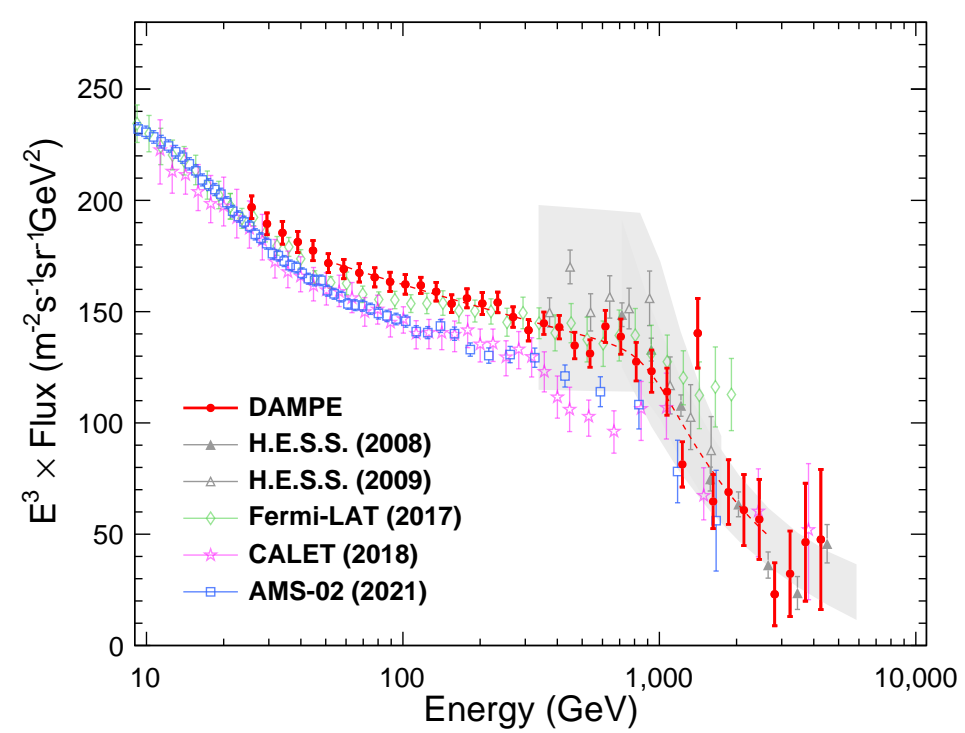

Figure 4: The CRE spectra measured by DAMPE (red dots) [21], compared with results from AMS-02 [22], Fermi-LAT [23], CALET [24], and H.E.S.S. [25, 26]. Shaded band shows the systematic uncertainties of the H.E.S.S. measurements.

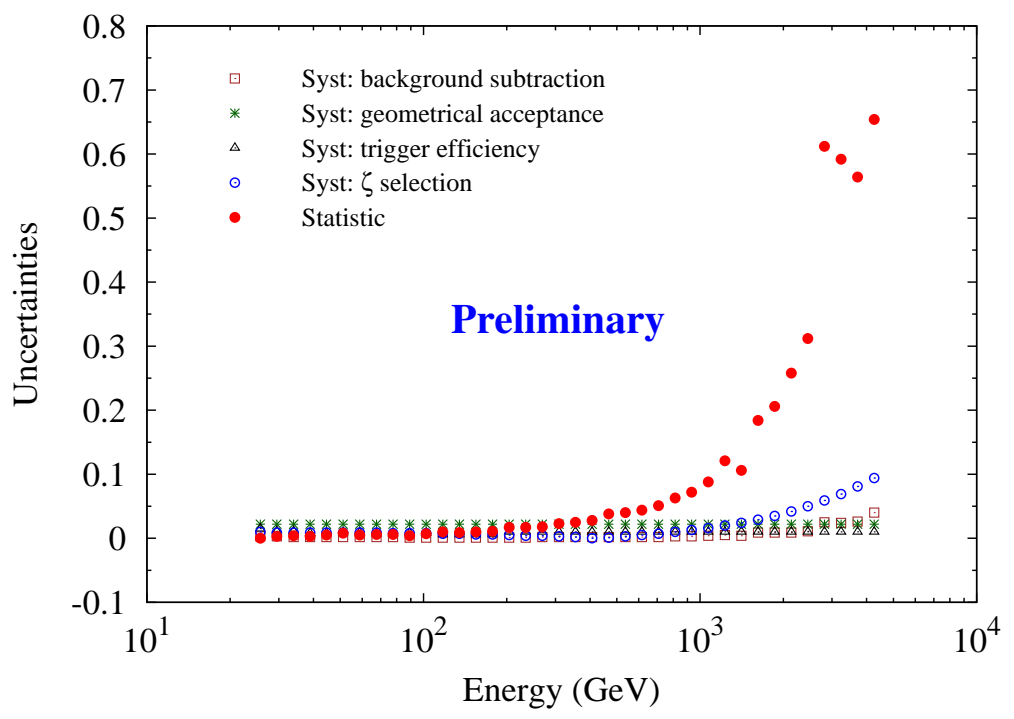

Figure 5: The components of measuring uncertainties for the DAMPE CRE spectrum [21].

Interestingly, these results show hints that the energy spectra of proton, Helium, and heavier nuclei soften at $\sim 10 \mathrm{TeV}$ energies [35, 37]. It is thus very important to have improved measurements with substantially higher statistics and better control of systematic uncertainties in the energy range between $\mathrm{TeV}$ and $100 \mathrm{TeV}$ to clarify the spectral behaviors of CRs. A relatively large acceptance and thick calorimeter (about 1.6 nuclear interaction lengths) of the DAMPE makes it appropriate for observations of $\mathrm{CR}$ nuclei.

After proper corrections such as the light attenuation correction, geometrical alignment and 
quenching-equalization [9-11, 38], the "PSD charge" distributions of selected events in the CR proton analysis with several deposit energy ranges are shown in Fig. 6, along with the MC simulation results. The Helium background is estimated according to a fitting to the charge distribution of the flight data using MC templates, which turns out to be $\lesssim 1 \%$ for deposited energies below $10 \mathrm{TeV}$ and up to $\sim 5 \%$ around $50 \mathrm{TeV}$. The electron background is found to be extremely small thanks to the high electron-proton discrimination capability of the DAMPE [21]. Results from similar work in the CR helium analysis are shown in Fig. 7. And some independent approaches of studying the CR helium are also being carried out [39].
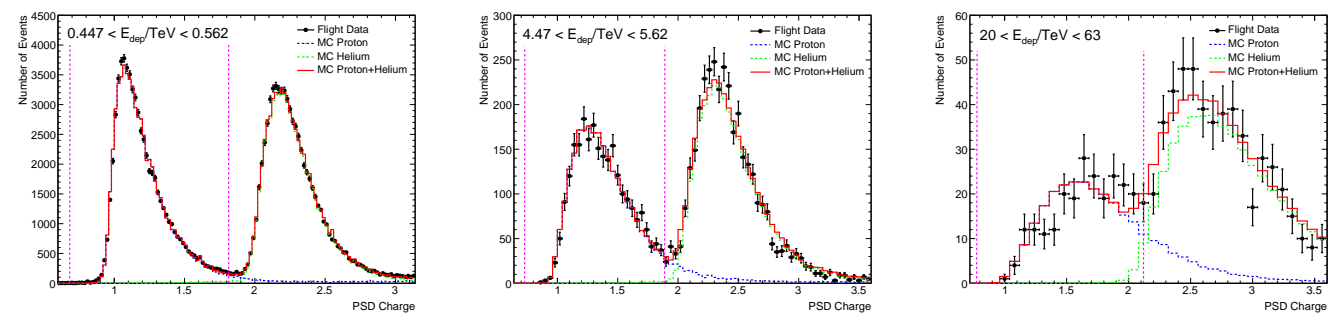

Figure 6: The PSD charge distribution during the CR proton analysis, compared with simulations of protons and helium nuclei. Plot from [40].
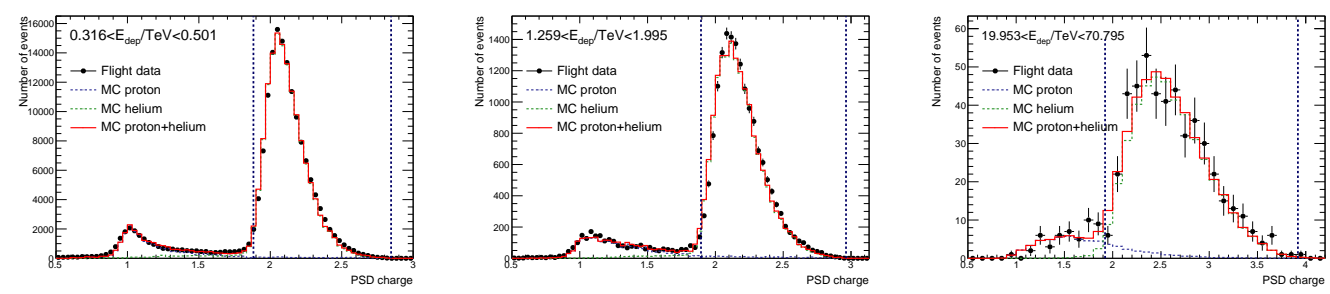

Figure 7: The PSD charge distribution during the CR Helium analysis, compared with simulations of protons and helium nuclei. Plot from [41].
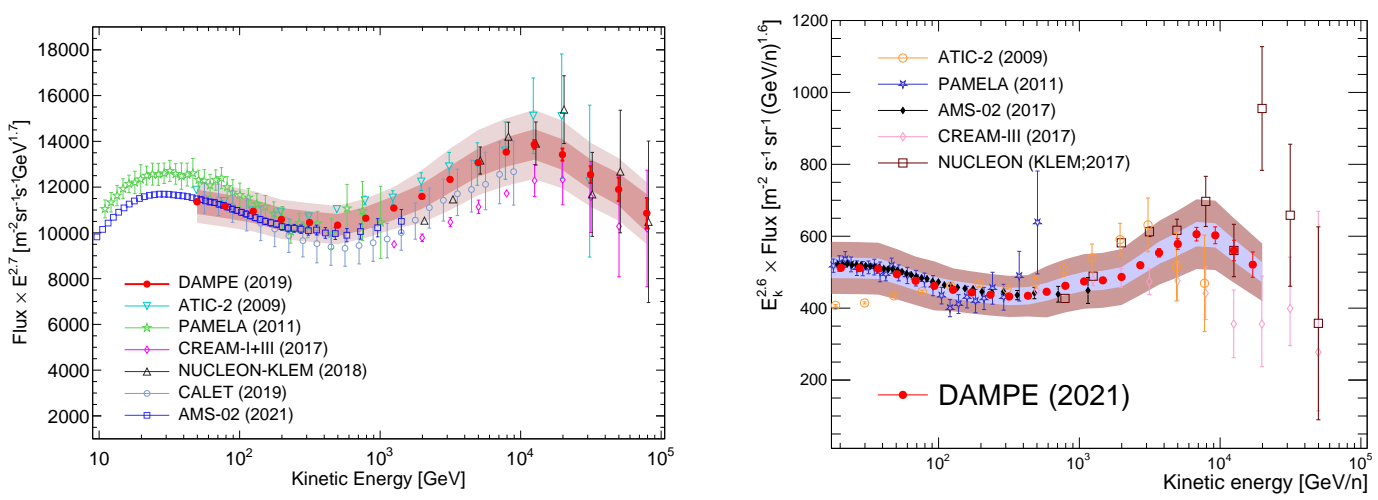

Figure 8: The proton and helium spectra measured by DAMPE (red dots; [40, 40]), compared with those measured by other experiments [22, 30, 33, 35-37, 44]. The total uncertainties including the statistical ones and the systematic ones are shown by the shaded bands. 
Using the first 30 months of DAMPE data, we analyze the proton component in CRs [40] (see Fig. 8 left). And with the first 4.5-year data, we analyze the CR Helium spectrum [41, 42] (see Fig. 8 right). Only events satisfying the high-energy trigger (HET) are selected. The major uncertainty comes from the systematical uncertainty by different hadronic models used in the Monte Carlo simulations [43].

The DAMPE results of CR proton and helium confirm the spectral hardenings at hundreds of $\mathrm{GeV} / \mathrm{n}$ revealed previously [22, 30, 33, 35-37, 44], and most importantly, reveals a mutual spectral softening around $10 \mathrm{TeV} / \mathrm{n}$ in both proton and helium spectra. A smoothly broken power-law fit gives that the spectral index changes from -2.60 to -2.85 at $13.6_{-4.8}^{+4.1} \mathrm{TeV}$ for proton and from -2.41 to -2.92 at $34.4_{-9.8}^{+6.7} \mathrm{TeV}$ for helium. These softenings feature may have very interesting implication in modeling the origin and propagation of CRs (e.g., [45-47]).

The boron to carbon flux ratio directly indicates the average amount of interstellar material traversed by cosmic rays [48]. Over the last 20 years, there have been many measurements on it [49-52]. Fig. 9 shows the preliminary result on the measurement of $B / C$ flux ratio [53], which is consistent with results from other experiments at lower energies. The analysis up to $4 \mathrm{TeV} / \mathrm{n}$ is still on going.

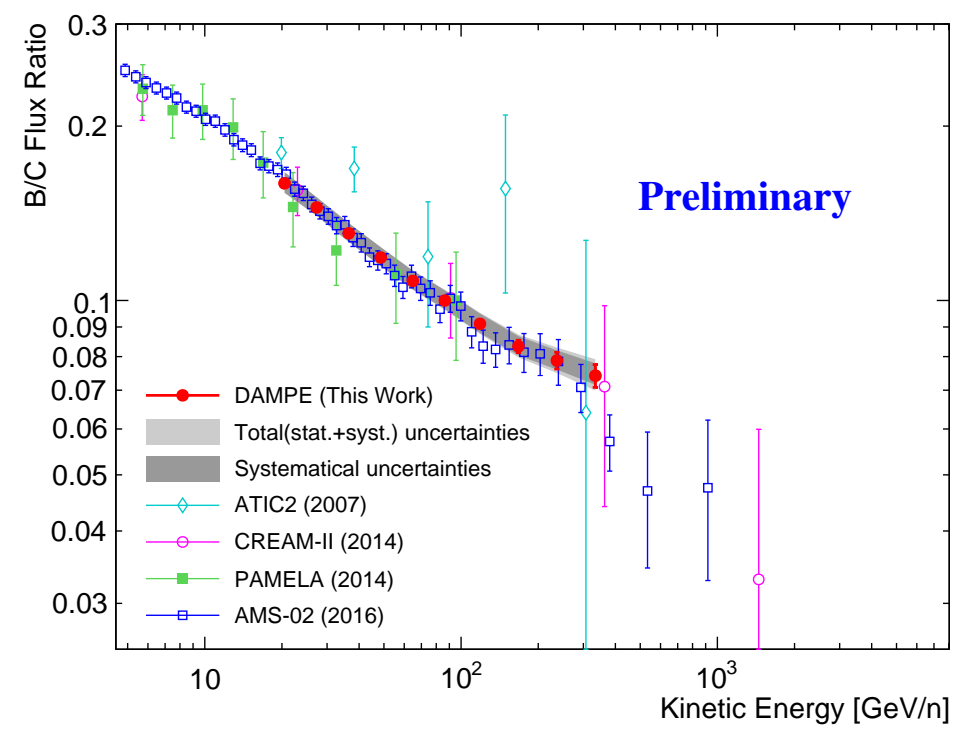

Figure 9: Preliminary measurement of the boron to carbon flux ratio by DAMPE. Plot from [53].

The analyses of other CR nuclei are being carried out, such as the light component (proton plus helium) spectrum [54], the carbon and oxygen fluxes [55], the iron spectrum [56], etc. The massive Monte Carlo simulation plays a very important role in the nuclei analyses [57].

\section{Cosmic ray anisotopies}

Cosmic rays propagate diffusively in the turbulent magnetic fields of the Milky Way, which results in nearly isotropy of their arrival directions in the sky. However, tiny anisotropies were observed (e.g., [58]), reflecting important properties of the origin and propagation of cosmic rays. 
Currently the detection of the anisotropies is mostly from ground or underground experiments, which have limited composition resolution and sky coverage. Furthermore, the ground-based air shower experiments also suffer from problems of calibration of the absolute efficiencies for different latitude bands. Therefore it is very important to measure the anisotropies from space direct detection experiments.

There were efforts to search for anisotropies of either CR nuclei or electrons/positrons by PAMELA [59], Fermi-LAT [60, 61], and AMS-02 [62]. No significant anisotropies were detected except that PAMELA reported a potential detection of a dipole anisotropy using its calorimeter sample of all CRs [63].

Using five years of the DAMPE data, we study the anisotropies of all CRs [64]. After removing events recorded when the satellite is in the SAA region, we further select the events with maximum zenith angles of $45^{\circ}$ in the detector coordinate and deposited energies above $100 \mathrm{GeV}$ in the BGO calorimeter. Note that the primary events should have energies higher by a factor of $2 \sim 3$ in this energy range. After all these cuts, we get a total number of events of about $4.44 \times 10^{7}$.
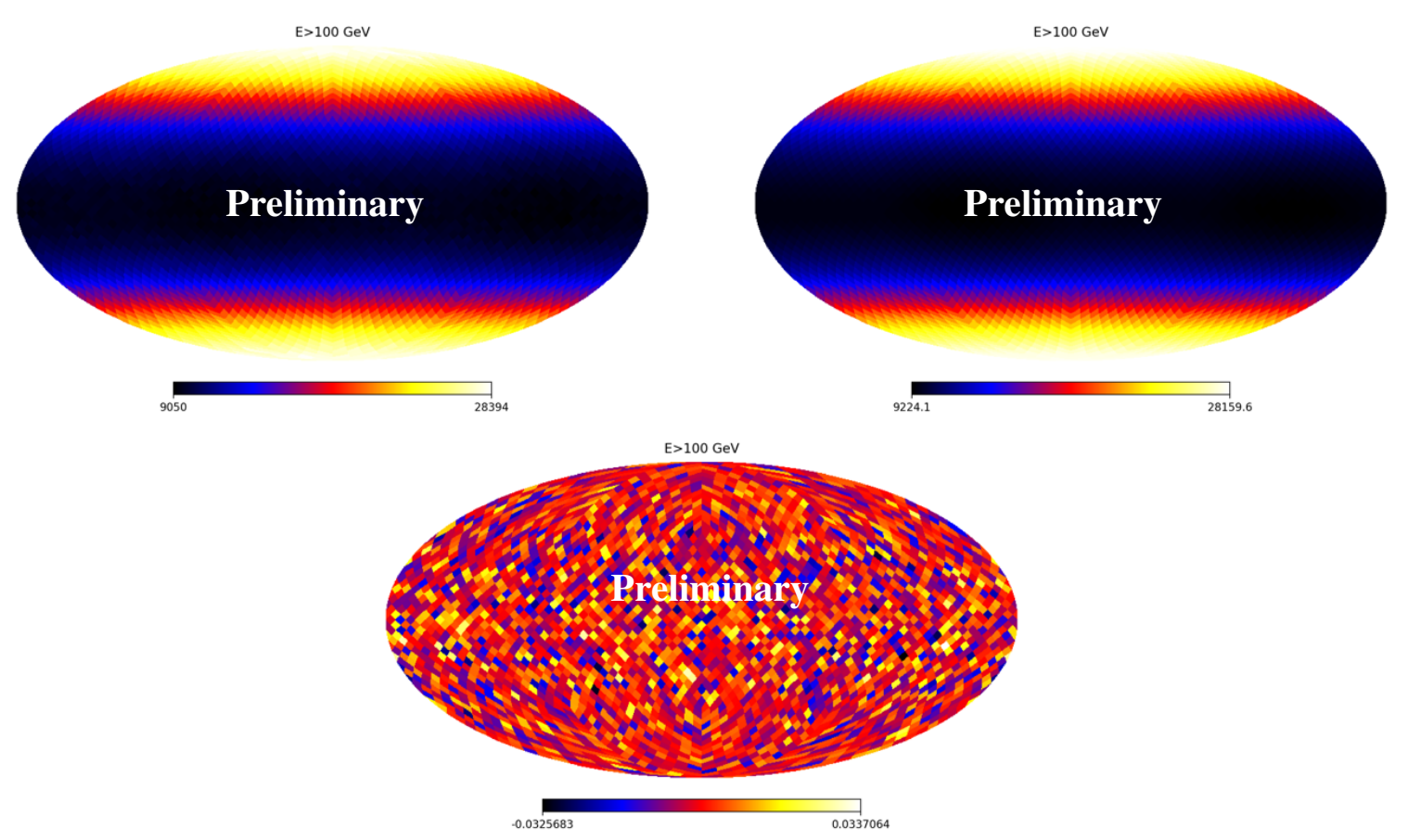

Figure 10: Skymaps of DAMPE raw events (top-left panel), the reference exposure map from the rate-based method (top-right panel), and the relative intensity map (bottom panel). Plot from [64].

The skymap based on the selected events in the equatorial coordinate is shown in the top-left panel of Fig. 10. However, the exposure of the DAMPE is highly non-uniform. To obtain a precise exposure map is crucial to the search for tiny anisotropies of CR arrival directions. The reference exposure map is created with the rate-based method proposed in [60]. An average of 100 reference maps is shown in the top-right panel of Fig. 10. The relative intensity map, defined as $\delta I=D / R-1$, where $D$ is the data map and $R$ is the reference map, is shown in the bottom panel. The result is consistent with the null-hypothesis. The $95 \%$ confidence level upper limit of the dipole amplitude 
is $\delta_{\mathrm{UL}}=1.2 \times 10^{-3}$ for five-year data sample. Further improvement of the sensitivity of anisotropy searches can be expected with more data added.

\section{6. $\gamma$-ray observations}

Gamma-ray astronomy is one of the three major scientific objectives of the DAMPE. Thanks to its unprecedented high energy resolution, the DAMPE is expected to play a key role in searching for monochromatic $\gamma$-ray line emission. The observations of $\gamma$-ray transients are also very interesting and important in the multi-messenger era, particularly in the case that gravitational waves [65] and very-high-energy astrophysical neutrinos [66] have been discovered.

Compared with charged CRs, the flux of $\gamma$-rays is significantly smaller by a factor of $10^{5} \sim 10^{6}$. An effect way to pick out $\gamma$-rays with little contamination of charged CRs is the key for studies of the $\gamma$-ray astronomy. A dedicated algorithm has been developed to resolve $\gamma$-rays from the flight data [12]. It includes joint efforts of electromagnetic and hadronic shower discrimination with the BGO calorimeter and the veto of charged particles with the PSD. With such a method, we can reach an effective acceptance of $\sim 0.19(0.11) \mathrm{m}^{2} \mathrm{sr}$ at $10\left(10^{3}\right) \mathrm{GeV}$ of $\gamma$-ray detection, with a purity of $\sim 99 \%$ for energies higher than $5 \mathrm{GeV}$ [12]. Making use of the selected photons, we resolve more than $200 \gamma$-ray point sources from the five-year DAMPE data [67].

We perform the search for $\gamma$-ray line structures using five years of DAMPE data [68]. The scientific tool of DmpST and the embedded instrumental response functions (IRFs) based on MC simulations have been used [70]. To improve the sensitivity, we develop two types of dedicated data sets $[68,69]$ and adopt the signal-to-noise ratio optimized regions of interest (ROIs) for different dark matter density profiles and different types of dark matter (annihilating or decaying). No line signals or candidates are found between $10 \mathrm{GeV}$ and $300 \mathrm{GeV}$ in the Galaxy. The constraints on the velocity-averaged cross section for $\chi \chi \rightarrow \gamma \gamma$ and the decay lifetime for $\chi \rightarrow \gamma v$, both at 95\% confidence level, have been calculated and the systematic uncertainties have been taken into account. Comparing to the previous 5.8 year results of Fermi-LAT [71], though DAMPE has an acceptance smaller by a factor of $\sim 10$, similar constraints on the DM parameters are achieved and below $100 \mathrm{GeV}$ the lower limits on the decay lifetime are even stronger by a factor of a few. Our results demonstrate the potential of high-energy-resolution observations on dark matter detection.

Fermi bubbles, first discovered using the Fermi-LAT data, consist of two large bubbles, each of which is approximately $40^{\circ}$ wide and extends to $55^{\circ}$ above and below the Galactic center [72, 73]. We use 4.8-year DAMPE photon data to analyze their emission. The significance of the FBs as a whole is found to be $\sim 18.0 \sigma$ if the standard GDE model is adopted. The spectrum is well consistent with that from the Fermi-LAT [73]. More details can be found in [74].

\section{Other studies}

The DAMPE data can also be used for studies on solar activities. We study the impact of a big solar flare occurred in September 2017 on the low energy CRE fluxes, and observe the Forbush decreases in the DAMPE data [75]. Both the amplitude and recovery time of fluxes of CRE show clear energy-dependence, which is important in probing the disturbances of the interplanetary environment by the coronal mass ejections. 

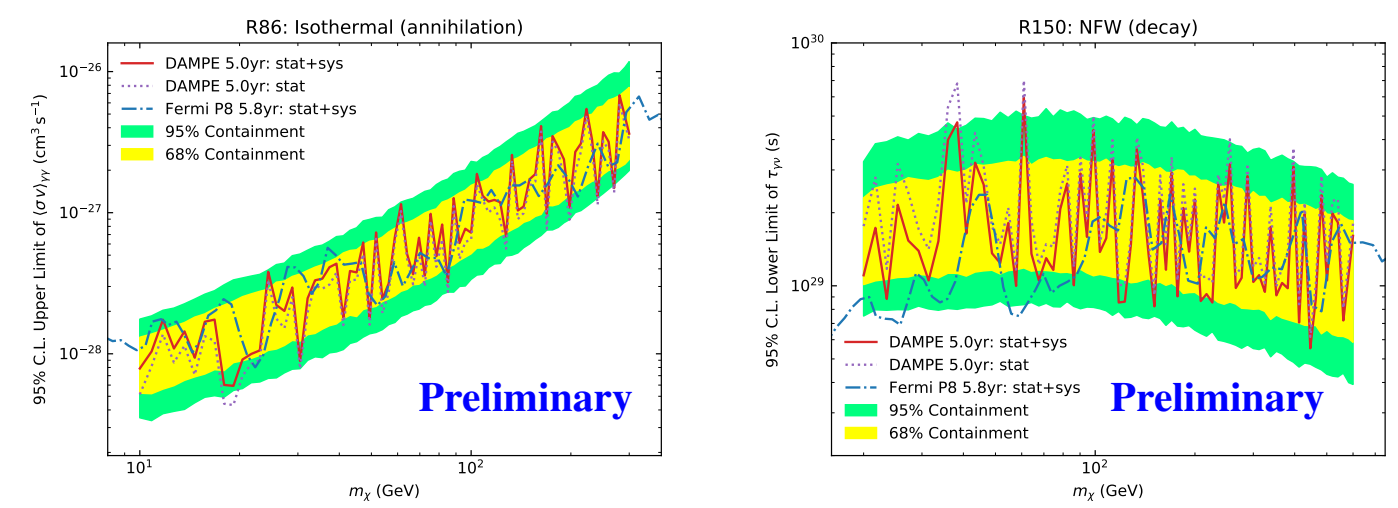

Figure 11: The constraints for annihilating and decaying dark matter. The left panel shows the $\langle\sigma v\rangle_{\gamma \gamma}$ upper limits of annihilating DM assuming the Isothermal profile. The right panel presents the $\tau_{\gamma v}$ lower limit of decaying DM assuming the NFW profile. Yellow (green) bands show the 68\% (95\%) expected containment obtained from 1000 simulations of background emission with systematic uncertainties involved. The red solid and purple dotted lines are the results with and without the systematic uncertainties respectively. The blue dot-dashed lines show the 5.8-year Fermi-LAT constraints [71]. Plot from [68].

Quarks are established as fundamental constituents of hadrons in the standard model of particle physics. They have fractional charges of $1 / 3$ or $2 / 3$, but do not appear individually according to the quark confinement theory. Observations of fraction charge particles are important in probing new physics beyond the standard model. We use MIP trigger events to do such searches of fractional charge particles. The analysis method and progress can be found in [76].

\section{Conclusion}

The DAMPE mission has operated in space very smoothly since its launch. The detector works continuously and stably, with over 10 billion CR events being recorded in its 5.5 year run. Careful calibration using the flight data has been made, which verifies the designed performance of the instrument and ensures good measurements of the charge, direction, energy, and type of incident particles. Those physical quantities are the basis of the science analysis.

Precise measurements of the cosmic ray electrons/positions, nuclei and $\gamma$-rays have been performed. The physical results of DAMPE are shedding new light on the understanding of the dark matter, cosmic ray physics and gamma-ray astronomy. More analyses are being carried out along with the data accumulating, and we expect more results from DAMPE.

\section{Acknowledgements}

The DAMPE mission is funded by the strategic priority science and technology projects in space science of Chinese Academy of Sciences. In China the data analysis is supported in part by the National Key Research and Development Program of China (No. 2016YFA0400200), the National Natural Science Foundation of China (Nos. 11921003, U1738205, U1738206, U1738207, U1738208, U1738210, 12022302, 11773086, 12173099, 12003074, 11903084, 11622327, U1738123, U1738138), 
the Youth Innovation Promotion Association CAS, and the Entrepreneurship and Innovation Program of Jiangsu Province. In Europe the activities and data analysis are supported by the Swiss National Science Foundation (SNSF), Switzerland, the National Institute for Nuclear Physics (INFN), Italy.

This proceeding is more or less an update of [77], sharing a similar structure and part of similar statements.

\section{References}

[1] J. Chang, Dark Matter Particle Explorer: The First Chinese Cosmic Ray and Hard Gamma-ray Detector in Space, Chinese Journal of Space Science 34 (2014) 550.

[2] J. Chang et al. [DAMPE Collaboration], The DArk Matter Particle Explorer mission, Astropart. Phys. 95 (2017) 6.

[3] Y. Yu et al., The plastic scintillator detector for DAMPE, Astropart. Phys. 94 (2017) 1.

[4] P. Azzarello et al., The DAMPE silicon-tungsten tracker, Nucl. Instrum. Methods Phys. Res. A 831 (2016) 378.

[5] Z. Zhang et al., Design of a high dynamic range photomultiplier base board for the BGO ECAL of DAMPE, Nucl. Instrum. Methods Phys. Res. A 780 (2015) 21.

[6] M. He et al., GEANT4 Simulation of Neutron Detector for DAMPE, Acta Astron. Sin. 57 (2016) 1.

[7] G. Ambrosi et al. [DAMPE Collaboration], The on-orbit calibration of DArk Matter Particle Explorer, Astropart. Phys. 106 (2019) 18.

[8] Y. L. Zhang et al., The status of DAMPE satellite in space, Proc. Sci. , PoS(ICRC2019)166 (2019).

[9] M. Ding et al., Calibration of the DAMPE Plastic Scintillator Detector and its on-orbit performance, Res. Astron. Astrophys. 19 (2019) 047.

[10] P. X. Ma et al., A Method of Alignment of the Plastic Scintillator Detector of DAMPE, Res. Astron. Astrophys. 19 (2019) 082.

[11] T. K. Dong et al., Charge measurement of cosmic ray nuclei with the plastic scintillator detector of DAMPE, Astropart. Phys. 105 (2019) 31.

[12] Z. L. Xu et al., An algorithm to resolve $\gamma$-rays from charged cosmic rays with DAMPE, Res. Astron. Astrophys. 18 (2018) 027.

[13] A. Tykhonov et al., Internal alignment and position resolution of the silicon tracker of DAMPE determined with orbit data, Nucl. Instrum. Meth. A 893 (2018) 43.

[14] C. Perrina et al., Performance of the DAMPE silicon-tungsten tracker during the first 5 years of in-orbit operation, Proc. Sci. , PoS(ICRC2021)084 (2021). 
[15] A. Ruina et al., Charge Loss Correction in the Silicon-Tungsten Tracker-Converter for ProtonHelium Charge Identification in the DAMPE Detector, Proc. Sci. , PoS(ICRC2021)083 (2021).

[16] Y. Wei et al., On-orbit performance of the DAMPE BGO calorimeter, Proc. Sci. , PoS(ICRC2021)081 (2021).

[17] Z. Y. Zhang et al., The calibration and electron energy reconstruction of the BGO ECAL of the DAMPE detector, Nucl. Instrum. Meth. A 836 (2016) 98.

[18] W. Jiang et al., Determination of the South Atlantic Anomaly from DAMPE data, Proc. Sci. , PoS(ICRC2017)228 (2017).

[19] W. Jiang et al., The boresight alignment with the DArk Matter Particle Explorer, Res. Astron. Astrophys. 20, (2020) 092

[20] J. Chang et al., Resolving electrons from protons in ATIC, Adv. Space Res. 42 (2008) 431.

[21] G. Ambrosi et al. [DAMPE Collaboration], Direct detection of a break in the teraelectronvolt cosmic-ray spectrum of electrons and positrons, Nature 552 (2017) 63.

[22] M. Aguilar et al. [AMS Collaboration], The Alpha Magnetic Spectrometer (AMS) on the international space station: Part II - Results from the first seven years, Phys. Rept. 894, (2021) 1 .

[23] S. Abdollahi et al. [Fermi-LAT Collaboration], Cosmic-ray electron-positron spectrum from $7 \mathrm{GeV}$ to $2 \mathrm{TeV}$ with the Fermi Large Area Telescope, Phys. Rev. D 95 (2017) 082007.

[24] O. Adriani et al. [CALET Collaboration], Extended Measurement of the Cosmic-Ray Electron and Positron Spectrum from $11 \mathrm{GeV}$ to $4.8 \mathrm{TeV}$ with the Calorimetric Electron Telescope on the International Space Station, Phys. Rev. Lett. 120, (2018) 261102.

[25] F. Aharonian et al. [H.E.S.S. Collaboration], The energy spectrum of cosmic-ray electrons at TeV energies, Phys. Rev. Lett. 101 (2008) 261104 [arXiv:0811.3894].

[26] F. Aharonian et al. [H.E.S.S. Collaboration], Probing the ATIC peak in the cosmic-ray electron spectrum with H.E.S.S, Astron. Astrophys. 508 (2009) 561 [arXiv:0905.0105].

[27] Q. Yuan et al., Interpretations of the DAMPE electron data, arXiv:1711.10989.

[28] Q. Yuan and L. Feng, Dark Matter Particle Explorer observations of high-energy cosmic ray electrons plus positrons and their physical implications, Sci. China Phys. Mech. Astron. 61 (2018) 101002.

[29] K. Fang, X. J. Bi and P. F. Yin, Explanation of the knee-like feature in the DAMPE cosmic $e^{-}+e^{+}$energy spectrum, Astrophys. J. 854 (2018) 57.

[30] O. Adriani et al. [PAMELA Collaboration], PAMELA Measurements of Cosmic-ray Proton and Helium Spectra, Science 332 (2011) 69. 
[31] M. Aguilar et al. [AMS Collaboration], Precision Measurement of the Proton Flux in Primary Cosmic Rays from Rigidity 1 GV to 1.8 TV with the Alpha Magnetic Spectrometer on the International Space Station, Phys. Rev. Lett. 114 (2015) 171103.

[32] M. Aguilar et al. [AMS Collaboration], Precision Measurement of the Helium Flux in Primary Cosmic Rays of Rigidities 1.9 GV to 3 TV with the Alpha Magnetic Spectrometer on the International Space Station, Phys. Rev. Lett. 115, (2015) 211101.

[33] A. D. Panov et al., Energy Spectra of Abundant Nuclei of Primary Cosmic Rays from the Data of ATIC-2 Experiment: Final Results, Bull. Russ. Acad. Sci. Phys. 73 (2009) 564.

[34] H. S. Ahn et al., Discrepant hardening observed in cosmic-ray elemental spectra, Astrophys. J. 714 (2010) L89.

[35] Y. S. Yoon et al., Proton and Helium Spectra from the CREAM-III Flight, Astrophys. J. 839 (2017) 5 .

[36] E. Atkin et al. [NUCLEON Collaboration], First results of the cosmic ray NUCLEON experiment, JCAP 07 (2017) 020.

[37] E. Atkin et al., New Universal Cosmic-Ray Knee near a Magnetic Rigidity of $10 \mathrm{TV}$ with the NUCLEON Space Observatory, JETP Lett. 108 (2018) 5.

[38] P. X. Ma et al., Charge measurement of cosmic rays by Plastic Scintillator Detector of DAMPE, Proc. Sci. , PoS(ICRC2021)073 (2021).

[39] M. Stolpovskiy et al., Machine learning methods for helium flux analysis with DAMPE experiment, Proc. Sci. , PoS(ICRC2021)077 (2021).

[40] Q. An et al. [DAMPE Collaboration], emphMeasurement of the cosmic-ray proton spectrum from $40 \mathrm{GeV}$ to $100 \mathrm{TeV}$ with the DAMPE satellite, Sci. Adv. 5 (2019) eaax3793

[41] F. Alemanno et al. [DAMPE Collaboration], Measurement of the cosmic ray helium energy spectrum from $70 \mathrm{GeV}$ to $80 \mathrm{TeV}$ with the DAMPE space mission, Phys. Rev. Lett. 126 (2021) 201102.

[42] M. Di Santo et al., Cosmic Ray Helium spectrum measured by the DAMPE experiment, Proc. Sci. , PoS(ICRC2021)114 (2021).

[43] W. Jiang et al., Comparison of Proton Shower Developments in the BGO Calorimeter of the Dark Matter Particle Explorer between GEANT4 and FLUKA Simulations, Chin. Phys. Lett. 37 (2020) 119601.

[44] O. Adriani et al. [CALET Collaboration], Direct Measurement of the Cosmic-Ray Proton Spectrum from $50 \mathrm{GeV}$ to $10 \mathrm{TeV}$ with the Calorimetric Electron Telescope on the International Space Station, Phys. Rev. Lett. 122 (2019) 181102.

[45] V. I. Zatsepin and N. V. Sokolskaya, Three component model of cosmic ray spectra from 100-gev up to 100-pev, Astron. Astrophys. 458 (2006) 1. 
[46] W. Liu, Y. Q. Guo and Q. Yuan, Indication of nearby source signatures of cosmic rays from energy spectra and anisotropies, JCAP 10 (2019) 010.

[47] C. Yue et al., Implications on the origin of cosmic rays in light of $10 \mathrm{TV}$ spectral softenings, Front. Phys. 15 (2020) 24601.

[48] A. W. Strong, I. V. Moskalenko and V. S. Ptuskin, Cosmic-ray propagation and interactions in the Galaxy, Ann. Rev. Nucl. Part. Sci. 57 (2007) 285.

[49] A. D. Panov et al., Relative abundances of cosmic ray nuclei $B-C-N-O$ in the energy region from $10 \mathrm{GeV} / \mathrm{n}$ to $300 \mathrm{GeV} / \mathrm{n}$. Results from ATIC-2 (the science flight of ATIC), arXiv:0707.4415.

[50] H. S. Ahn et al., Measurements of cosmic-ray secondary nuclei at high energies with the first flight of the CREAM balloon-borne experiment, Astropart. Phys. 30 (2008) 133.

[51] O. Adriani et al., Measurement of boron and carbon fluxes in cosmic rays with the PAMELA experiment, Astrophys. J. 791 (2014) 93.

[52] M. Aguilar et al. [AMS Colllaboration], Precision Measurement of the Boron to Carbon Flux Ratio in Cosmic Rays from 1.9 GV to 2.6 TV with the Alpha Magnetic Spectrometer on the International Space Station, Phys. Rev. Lett. 117 (2016) 231102.

[53] C. Yue et al., Measurement of the Boron to Carbon Flux Ratio in Cosmic Rays with the DAMPE Experiment, Proc. Sci. , PoS(ICRC2021)126 (2021).

[54] F. Alemanno et al., Measurement of the light component $(p+H e)$ energy spectrum with the DAMPE space mission, Proc. Sci. , PoS(ICRC2021)117 (2021).

[55] L. Wu et al., Towards the measurement of carbon and oxygen spectra in cosmic rays with DAMPE, Proc. Sci. , PoS(ICRC2021)128 (2021).

[56] Z. Xu et al., Direct Measurement of the Cosmic-Ray Iron Spectrum with the Dark Matter Particle Explorer, Proc. Sci. , PoS(ICRC2021)115 (2021).

[57] W. Jiang et al., Simulation of the DAMPE detector, Proc. Sci., PoS(ICRC2021)082 (2021).

[58] M. Amenomori [Tibet AS-gamma Collaboration], Anisotropy and Corotation of Galactic Cosmic Rays, Science 314 (2006) 439.

[59] O. Adriani et al., Search for Anisotropies in Cosmic-ray Positrons Detected by the PAMELA Experiment, Astrophys. J. 811 (2015) 21.

[60] S. Abdollahi et al. [Fermi-LAT Collaboration], Search for Cosmic-Ray Electron and Positron Anisotropies with Seven Years of Fermi Large Area Telescope Data, Phys. Rev. Lett. 118 (2017) 091103.

[61] M. Ajello et al. [Fermi-LAT Collaboration], A Search for Cosmic-ray Proton Anisotropy with the Fermi Large Area Telescope, Astrophys. J. 883 (2019) 33. 
[62] G. La Vacca [AMS-02 Collaboration], Search for Cosmic Ray Anisotropy with the Alpha Magnetic Spectrometer on the International Space Station, arXiv:1612.08957.

[63] O. Adriani et al. [PAMELA Collaboration], Ten years of PAMELA in space, Riv. Nuovo Cim. 40 (2017) 1.

[64] S. Lei et al., Studies of cosmic ray anisotropies with DAMPE, Proc. Sci. , PoS(ICRC2021)125 (2021).

[65] B. P. Abbott et al. [LIGO Scientific and Virgo Collaborations], Observation of Gravitational Waves from a Binary Black Hole Merger, Phys. Rev. Lett. 116 (2016) 061102.

[66] M. G. Aartsen et al. Multimessenger observations of a flaring blazar coincident with highenergy neutrino IceCube-170922A, Science 361 (2018) eaat1378.

[67] K. K. Duan et al., Observations of gamma-ray sources with DAMPE, Proc. Sci. , PoS(ICRC2021)631 (2021).

[68] Z. Xu et al., Search for gamma-ray lines in the Galaxy with DAMPE, Proc. Sci. , PoS(ICRC2021)632 (2021).

[69] Z. L. Xu et al., Optimal gamma-ray selections for monochromatic line searches with DAMPE, arXiv:2107.13208.

[70] K. K. Duan et al., DmpIRFs and DmpST: DAMPE Instrument Response Functions and Science Tools for Gamma-Ray Data Analysis, Res. Astron. Astrophys. 19 (2019) 132.

[71] M. Ackermann et al. [Fermi-LAT Collaboration], Updated search for spectral lines from Galactic dark matter interactions with pass 8 data from the Fermi Large Area Telescope, Phys. Rev. D 91 (2015) 122002.

[72] M. Su, T. R. Slatyer and D. P. Finkbeiner, Giant Gamma-ray Bubbles from Fermi-LAT: AGN Activity or Bipolar Galactic Wind?, Astrophys. J. 724 (2010) 1044.

[73] M. Ackermann et al. [Fermi-LAT Collaboration], The Spectrum and Morphology of the Fermi Bubbles, Astrophys. J. 793 (2014) 64.

[74] Z. Q. Shen et al., Analyzing the Fermi Bubbles with DArk Matter Particle Explorer, Proc. Sci. , PoS(ICRC2021)640 (2021).

[75] F. Alemanno et al. [DAMPE Collaboration], Observations of Forbush Decreases of CosmicRay Electrons and Positrons with the Dark Matter Particle Explorer, Astrophys. J. Lett. 920 (2021) L43.

[76] C. Liu et al., Searching for fractionally charged particles based on DAMPE, Proc. Sci. , PoS(ICRC2021)130 (2021).

[77] Q. Yuan [DAMPE Collaboration], Progresses of the Dark Matter Particle Explorer experiment, Proc. Sci. , PoS(ICRC2019)017 (2019). 


\section{Full Authors List: DAMPE Collaboration}

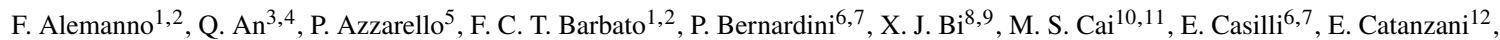
J. Chang ${ }^{10,11}$, D. Y. Chen ${ }^{10}$, J. L. Chen ${ }^{13}$, Z. F. Chen ${ }^{10,11}$, M. Y. Cui ${ }^{10}$, T. S. Cui ${ }^{14}$, Y. X. Cui ${ }^{10,11}$, H. T. Dai ${ }^{3,4}$, A. De Benedittis ${ }^{6,7}$, I. De Mitri ${ }^{1,2}$, F. de Palma ${ }^{6,7}$, M. Deliyergiyev ${ }^{5}$, M. Di Santo ${ }^{1,2}$, Q. Ding ${ }^{10,11}$, T. K. Dong ${ }^{10}$, Z. X. Dong ${ }^{14}$, G. Donvito ${ }^{15}$, D. Droz ${ }^{5}$, J.

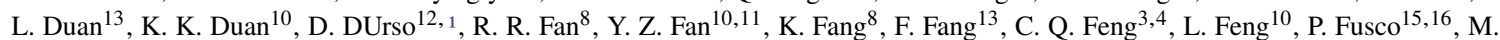

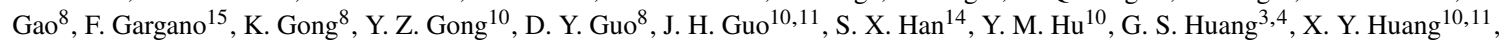

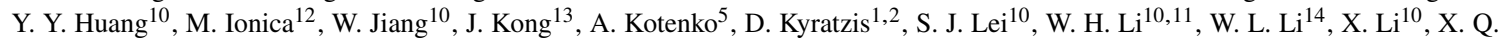

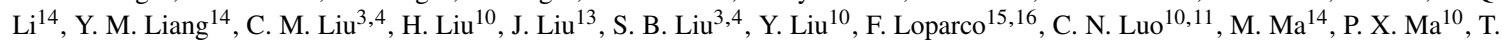

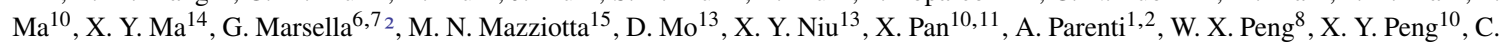
Perrina $^{5,3}$, R. Qiao ${ }^{8}$, J. N. Rao ${ }^{14}$, A. Ruina ${ }^{5}$, M. M. Salinas ${ }^{5}$, G. Z. Shang ${ }^{14}$, W. H. Shen ${ }^{14}$, Z. Q. Shen ${ }^{10}$, Z. T. Shen ${ }^{3,4}$, L. Silveri ${ }^{1,2}$, J. X. Song ${ }^{14}$, M. Stolpovskiy ${ }^{5}$, H. Su ${ }^{13}$, M. Su${ }^{17}$, H. R. Sun ${ }^{3,4}$, Z. Y. Sun ${ }^{13}$, A. Surdo ${ }^{7}$, X. J. Teng ${ }^{14}$, A. Tykhonov ${ }^{5}$, H. Wang ${ }^{14}$, J. Z.

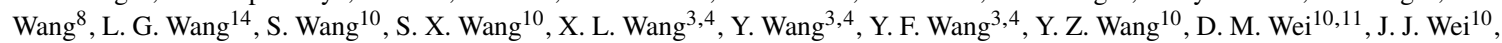
Y. F. Wei ${ }^{3,4}$, D. $\mathrm{Wu}^{8}$, J. Wu ${ }^{10,11}$, L. B. $\mathrm{Wu}^{1,2}$, S. S. $\mathrm{Wu}^{14}$, X. Wu ${ }^{5}$, Z. Q. Xia ${ }^{10}$, H. T. Xu ${ }^{14}$, Z. H. Xu ${ }^{10,11}$, Z. L. Xu ${ }^{10}$, E. H. Xu ${ }^{3,4}$, Z. Z. Xu ${ }^{3,4}$, G. F. Xue ${ }^{14}$, H. B. Yang ${ }^{13}$, P. Yang ${ }^{13}$, Y. Q. Yang ${ }^{13}$, H. J. Yao ${ }^{13}$, Y. H. Yu ${ }^{13}$, G. W. Yuan ${ }^{10,11}$, Q. Yuan ${ }^{10,11}$, C. Yue ${ }^{10}$, J. J. Zang $^{10^{4}}$ S. X. Zhang ${ }^{13}$, W. Z. Zhang ${ }^{14}$, Yan Zhang ${ }^{10}$, Yi Zhang ${ }^{10,11}$, Y. J. Zhang ${ }^{13}$, Y. L. Zhang ${ }^{3,4}$, Y. P. Zhang ${ }^{13}$, Y. Q. Zhang ${ }^{10}$, Z. Zhang ${ }^{10}$, Z. Y. Zhang ${ }^{3,4}$, C. Zhao ${ }^{3,4}$, H. Y. Zhao ${ }^{13}$, X. F. Zhao ${ }^{14}$, C. Y. Zhou ${ }^{14}$, and Y. Zhu ${ }^{14}$

${ }^{1}$ Gran Sasso Science Institute (GSSI), Via Iacobucci 2, I-67100 LAquila, Italy

${ }^{2}$ Istituto Nazionale di Fisica Nucleare (INFN) -Laboratori Nazionali del Gran Sasso, I-67100 Assergi, LAquila, Italy

${ }^{3}$ State Key Laboratory of Particle Detection and Electronics, University of Science and Technology of China, Hefei 230026, China

${ }^{4}$ Department of Modern Physics, University of Science and Technology of China, Hefei 230026, China

${ }^{5}$ Department of Nuclear and Particle Physics, University of Geneva, CH-1211, Switzerland

${ }^{6}$ Dipartimento di Matematica e Fisica E. De Giorgi, Università del Salento, I-73100, Lecce, Italy

${ }^{7}$ Istituto Nazionale di Fisica Nucleare (INFN) - Sezione di Lecce, I-73100, Lecce, Italy

${ }^{8}$ Institute of High Energy Physics, Chinese Academy of Sciences, Yuquan Road 19B, Beijing 100049, China

${ }^{9}$ University of Chinese Academy of Sciences, Yuquan Road 19A, Beijing 100049, China

${ }^{10}$ Key Laboratory of Dark Matter and Space Astronomy, Purple Mountain Observatory, Chinese Academy of Sciences, Nanjing 210023,

China

${ }^{11}$ School of Astronomy and Space Science, University of Science and Technology of China, Hefei 230026, China

${ }^{12}$ Istituto Nazionale di Fisica Nucleare (INFN) - Sezione di Perugia, I-06123 Perugia, Italy

${ }^{13}$ Institute of Modern Physics, Chinese Academy of Sciences, Nanchang Road 509, Lanzhou 730000, China

${ }^{14}$ National Space Science Center, Chinese Academy of Sciences, Nanertiao 1, Zhongguancun, Haidian district, Beijing 100190, China

${ }^{15}$ Istituto Nazionale di Fisica Nucleare (INFN) - Sezione di Bari, I-70125, Bari, Italy

${ }^{16}$ Dipartimento di Fisica M. Merlin dellUniversità e del Politecnico di Bari, I-70126, Bari, Italy

${ }^{17}$ Department of Physics and Laboratory for Space Research, the University of Hong Kong, Pok Fu Lam, Hong Kong SAR, China

${ }^{1}$ Now at Dipartimento di Chimica e Farmacia, Università di Sassari, I-07100, Sassari, Italy.

${ }^{2}$ Now at Dipartimento di Fisica e Chimica "E. Segrè”, Università degli Studi di Palermo, via delle Scienze ed. 17, I-90128 Palermo, Italy.

${ }^{3}$ Also at Institute of Physics, Ecole Polytechnique Federale de Lausanne (EPFL), CH-1015 Lausanne, Switzerland.

${ }^{4}$ Also at School of Physics and Electronic Engineering, Linyi University, Linyi 276000, China. 